\title{
Soluble mesothelin in effusions: a useful tool for the diagnosis of malignant mesothelioma
}

\author{
Jenette Creaney, Deborah Yeoman, Leanne K Naumoff, Michelle Hof, \\ Amanda Segal, Arthur William Musk, Nicholas De Klerk, \\ Nora Horick, Steven J Skates, Bruce W S Robinson
}

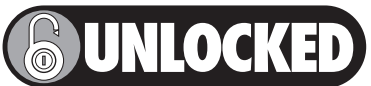

This paper is freely available online under the BMJ Journals unlocked scheme, see http://thorax.com/info/unlocked.dtl
See end of article for authors' affiliations

Correspondence to: Dr Jenette Creaney Department of Medicine and Pharmacology, University of Western Australia, Sir Charles Gairdner Hospital, Verdun Ave, Nedlands, 6009, Western Australia, Australia; creaneyj@cyllene. uwa.edu.au

Received 29 June 2006 Accepted 3 January 2007 Published Online First 13 March 2007

\begin{abstract}
Background: The diagnosis of malignant mesothelioma is frequently difficult, the most common differential diagnosis being reactive pleural conditions and metastatic adenocarcinoma. Soluble mesothelin levels in serum have recently been shown to be highly specific and moderately sensitive for mesothelioma. As most patients with mesothelioma present with exudative effusions of either the pleura or the peritoneum, a study was undertaken to determine if levels of mesothelin were raised in these fluids and if the increased levels could help to distinguish mesothelioma from other causes of exudative effusion.

Methods: Pleural fluid was collected from 192 patients who presented to respiratory clinics 152 with malignant mesothelioma, 56 with non-mesotheliomatous malignancies and 84 with effusions of nonneoplastic origin). Peritoneal fluid was collected from 42 patients 7 with mesothelioma, 14 with nonmesotheliomatous malignancies and 21 with benign effusions). Mesothelin levels were determined in effusion and serum samples by ELISA.

Results: Significantly higher levels of mesothelin were found in effusions of patients with mesothelioma; with a specificity of $98 \%$, the assay had a sensitivity of $67 \%$ comparing patients with mesothelioma and those with effusions of non-neoplastic origin. In 7 out of 10 cases mesothelin levels were raised in the effusion collected 3 weeks to 10 months before the diagnosis of mesothelioma was made; in 4 out of 8 of these, mesothelin levels were increased in the effusion but not in the serum.

Conclusions: Measurement of mesothelin concentrations in the pleural and/or peritoneal effusion of patients may aid in the differential diagnosis of mesothelioma in patients presenting with effusions.
\end{abstract}

M alignant mesothelioma is an aggressive, asbestosrelated tumour of the pleural and peritoneal cavities. Patients with pleural disease generally present with shortness of breath, and those with peritoneal disease with abdominal swelling. Both are usually associated with the presence of exudative effusions. Cytological diagnosis at presentation can be difficult, either because there are no malignant cells in the fluid or because the cells present are difficult to distinguish from reactive mesothelial cells or other malignant cells. ${ }^{1}$

Measurement of tumour markers in effusions has been reported to provide a complementary tool to aid in the diagnosis of the cause of an effusion. Differential levels of carcinoembryonic antigen (CEA), cancer antigen (CA) 15.3, CA72.4, CA19.9, CA549, neuron-specific enolase or cytokine fragment 19 (CYFRA 21-1) have been reported to differentiate between malignant and benign effusions. ${ }^{2-5}$ However, few data are available for the differential diagnosis of mesothelioma from other carcinomas. Low levels of CEA are found in effusions from patients with mesothelioma, and raised CEA levels provide a strong negative predictive value for this disease..$^{4-7}$ However, few studies have reported markers with a high positive predictive value for mesothelioma. Increased levels of CA15-3 have been reported in mesothelioma, ${ }^{3-5}$ and were found in one study to differentiate between mesothelioma and bronchial cancer. ${ }^{5}$ Higher levels of hyaluronic acid have been reported in effusions from patients with mesothelioma than those with other malignant diseases, but the difference was too small to be of diagnostic value. ${ }^{7}$ There is discrepancy in the literature on the ability of CYFRA 21-1 levels to differentiate between mesothelioma and other pleural malignancies..$^{78}$
Thus, there are currently no reliable tumour markers used on pleural or peritoneal exudates in routine clinical practice for assisting in the diagnosis of mesothelioma.

We recently reported a new serum tumour marker with high specificity and moderate sensitivity for mesothelioma-soluble mesothelin related protein-also known as mesothelin. Serum levels of these soluble mesothelin proteins were increased in $84 \%$ of patients with established mesothelioma and in only $2 \%$ of patients with other cancers or other lung or pleural disease. ${ }^{9}$ Similar findings were recently reported in a French study. ${ }^{10}$ The mesothelin protein itself is stable, with no significant difference in concentrations occurring following up to 10 freeze-thaw cycles, ${ }^{11}$ which also suggests that it may be useful as a clinical marker. The current study was undertaken to assess whether measurement of soluble mesothelin levels in effusions can provide additional diagnostic value to the existing conventional diagnostic tools.

\section{METHODS}

Patients and controls

From 1999 to 2005, serum, pleural and peritoneal effusions samples were collected from consecutive patients presenting at the respiratory clinics of either Sir Charles Gairdner Hospital or the Hollywood Specialist Centre in Perth, Western Australia. Effusions were obtained by routine pleurocentesis and were in excess to that required for diagnosis. The final diagnosis in all patients was confirmed by pathologists experienced in the diagnosis of effusions and included clinical follow-up of all

Abbreviations: $\mathrm{CA}$, cancer antigen; $\mathrm{CEA}$, carcinoembryonic antigen; CYFRA, cytokine fragment; ROC, receiver operating characteristic 
cases until death or for an average of 6.8 months (range 1-42) following the effusion to confirm that the clinical pattern matched the diagnosis. Effusions were classified as being malignant or non-malignant on the basis of cytological and immunohistochemical features, and malignant effusions were further classified as mesothelioma, metastatic adenocarcinoma or other malignancy. Non-malignant effusions were classified as exudates or transudates on the basis of Light's criteria. ${ }^{12}$ Effusions were classified as being associated with an infection if micro-organisms were detected in the fluid along with inflammatory cells or if the patient had pneumonia adjacent to the effusion.

Written and oral informed consent was obtained from all participants and the study was approved by the human ethics committees of Sir Charles Gairdner and Hollywood Hospitals.

\section{Measurement of mesothelin}

All specimens were received as fresh effusions with a volume range of 10-3000 $\mathrm{ml}$. Specimens were centrifuged for $10 \mathrm{~min}$ at $2000 \mathrm{~g}$ and the resulting supernatant was stored at $-80^{\circ} \mathrm{C}$ until assayed.

Soluble mesothelin concentrations were determined in duplicate following the manufacturer's instructions using a double determinant ELISA assay (Mesomark kit, Fujirebio Diagnostics, Malvern, Pennsylvania, USA). Mesothelin concentrations were determined from a standard curve performed on each plate and expressed as nM. Dilution of samples was carried out if necessary using the diluent supplied by the manufacturer. All assays were performed on coded samples by technical staff unaware of the patient's diagnosis.

\section{Immunolocalisations}

Mesothelin staining was examined in a subset of cases. Formalin-fixed paraffin-embedded cell-block specimens of pleural effusions from patients with mesothelioma were retrieved from PathWest Laboratories (Nedlands, Australia). Sections were deparaffinised with xylene and rehydrated in a graded series of ethanol. Antigen retrieval was performed using the pressure cooking method (3 min, 1 mM EDTA, $\mathrm{pH}$ 8.0). Before staining, endogenous peroxidase was blocked. Sections were processed by standard methods and incubated with antimesothelin antibody (clone 5B2, NovaCastra, Newcastle upon Tyne, UK; dilution 1:20) or anti-epithelial membrane antigen (clone E29, DakoCytomation, Glostrup, Denmark) for 60 min and washed in phosphate buffered saline (PBS). Immunodetection was performed using SuperPicTure Polymer detection reagent (Zymed, San Fransisco, California, USA). For negative controls, the primary antibody was omitted.

Staining was assessed by two independent observers (JC and AS). A positive result was defined as the presence of membranous stain in more than $20 \%$ of tumour cells. Staining intensity was graded semi-quantitatively as negative, weak $(1+)$, moderate $(2+)$ or strong $(3+)$.

\section{Statistical analysis}

Descriptive statistics and logistic regression analysis to predict cases from controls were performed using GraphPad Prism for Windows (GraphPad Software, San Diego, California, USA). Differences between groups of patients were assessed by Student's $t$ test after transforming mesothelin values to the log scale for which the distributions were closer to normality. For the same reason, median mesothelin values were estimated from the mean on the log scale and exponentiated to provide the estimate of the median on the original scale. All reported $p$ values are two-sided; a level of $\mathrm{p}<0.05$ was considered significant. Survival plots were generated by the KaplanMeier method and differences between patient groups were determined by the log-rank test. The significance of the mesothelin value nearest to the mesothelioma diagnosis for survival was assessed using Cox proportional hazards regression. ${ }^{13}$ Receiver operating characteristic (ROC) curves display the trade-off between sensitivity and specificity for mesothelin differentiating between groups of patients. Cross-validated ${ }^{14}$ estimates of sensitivity and specificity were obtained by the "leave-one-out" method to ameliorate over fitting bias using the Statistical Analysis System (SAS) Version 10 (SAS, Cary, North Carolina, USA) as the sample size was not sufficient to form independent training and validation data sets. Pearson's correlation coefficient was used to assess correlations between mesothelin values (log scale) in serum and pleural effusions.

\section{RESULTS}

\section{Characteristics of patients}

Pleural effusions were collected from 192 patients, of whom 52 were diagnosed with mesothelioma, 84 with effusions of nonneoplastic origin and 56 with non-mesotheliomatous malignancy (table 1). Of the 52 patients with mesothelioma, 15 were of epithelial histology, 5 biphasic and 9 sarcomatoid. In 23 cases a diagnosis was made on the basis of cytological findings..$^{15}$ in which case the histological subtype could not be confirmed. Peritoneal fluid and effusions were collected from 42 patients, of whom 7 were diagnosed with mesothelioma, 14 with nonmesotheliomatous malignancy, 6 with effusions of nonneoplastic origin and 15 with end-stage renal failure (table 1).

\section{Soluble mesothelin levels in pleural effusions}

Pleural effusions from patients with mesothelioma had significantly higher concentrations of mesothelin than those from patients with non-malignant effusions (median (SE) 27.7 (1.3) nM vs $4.1(0.8) \mathrm{nM}, \mathrm{p}<0.0001)$ of patients with nonmesothelioma malignancies (median (SE) 6.3 (1.2), p $<0.0001$; fig 1A, table 1).

Patients with mesothelioma with predominantly sarcomatoid histology had a significantly lower concentration of mesothelin in their effusions than patients with mesothelioma with a predominantly epithelial histology (median (SE) 46.9 (1.1) nM vs 4.5 (1.4) $\mathrm{nM}, \mathrm{p}<0.0001$, fig $1 \mathrm{~B}$, table 1 ). There was no significant difference in the levels of mesothelin in effusions from patients with sarcomatoid mesothelioma and those with non-malignant effusions.

As an indicator of the precision of the assay, control samples assayed in duplicate over 20 assays had a coefficient of variation of $5.3 \%$ for the high controls (mean (SD) mesothelin concentration $12.7(0.7) \mathrm{nM}$ ) and a coefficient of variation of $5.7 \%$ for the low controls (mean (SD) mesothelin concentration $4.4(0.3) \mathrm{nM})$.

ROC curves for mesothelin levels in effusions from different cohorts of patients showed that, compared with patients with non-malignant conditions, patients with mesothelioma (including those with the sarcomatoid variant) had an area under the curve of 0.898 (fig lC). At a cut-off value of $20 \mathrm{nM}$, the diagnostic specificity was $98 \%$ and the corresponding crossvalidated sensitivity in the combined histological groups of mesothelioma was $67 \%$. When the data were analysed excluding patients with mesothelioma of sarcomatoid histology, the area under the ROC curve was 0.964. At the same threshold value of $20 \mathrm{nM}$, the diagnostic specificity remained $98 \%$ and cross-validated sensitivity increased to $77 \%$. When the specificity was reduced to $90 \%$, the cross-validated sensitivity in this group was $86 \%$. At a cut-off value of $20 \mathrm{nM}, 8$ of the 56 patients with non-mesothelioma malignancies had increased levels of mesothelin in their effusions, giving a specificity of $86 \%$ for mesothelioma. 
Table 1 Patient characteristics and mesothelin values

\begin{tabular}{|c|c|c|c|}
\hline & $\begin{array}{l}\text { No of cases } \\
\text { (M/F) }\end{array}$ & $\begin{array}{l}\text { Median age, } \\
\text { years (range) }\end{array}$ & $\begin{array}{l}\text { Mean (SE) } \\
\text { mesothelin (nM) }\end{array}$ \\
\hline \multicolumn{4}{|l|}{ Pleural effusions } \\
\hline \multicolumn{4}{|l|}{ Mesothelioma } \\
\hline Epithelial & $12 / 3$ & $66.5(44-94)$ & $46.9(1.1)$ \\
\hline Biphasic & $4 / 1$ & $68(48-88)$ & $30.1(0.8)$ \\
\hline Sarcomatoid & $9 / 0$ & 77 (57-82) & 4.5 (1.4) \\
\hline Cytology only & $21 / 2$ & $68.5(44-89)$ & $39.2(1.0)$ \\
\hline All & 52 & $68.5(44-94)$ & $27.7(1.3)$ \\
\hline \multicolumn{4}{|l|}{ Non-malignant effusions } \\
\hline Transudate & $22 / 13$ & 75 (48-93) & $4(0.7)$ \\
\hline Exudate (non-infection) & $24 / 6$ & 75 (34-94) & $4.3(0.9)$ \\
\hline Exudate (infection) & $13 / 6$ & $72(34-91)$ & $4.3(0.9)$ \\
\hline All & 84 & $75(12-94)$ & $4.1(0.8)$ \\
\hline \multicolumn{4}{|c|}{ Non-mesothelioma malignant effusions } \\
\hline Lung cancer & 22 & $71(51-79)$ & $9.4(1.1)$ \\
\hline Non-small cell & $15 / 4$ & $70.5(51-79)$ & $10.4(1.1)$ \\
\hline Adenocarcinoma & $14 / 3$ & $65(51-79)$ & $11.8(1.1)$ \\
\hline Squamous cell & $1 / 0$ & 76 & 5.5 \\
\hline Large cell & $0 / 1$ & 71 & 1.8 \\
\hline Small cell & $3 / 0$ & 76.5 (74-79) & $4.8(0.6)$ \\
\hline Other cancer & 34 & $66(33-93)$ & $4.8(1.1)$ \\
\hline Colorectal & $2 / 3$ & $75(50-88)$ & $5(0.6)$ \\
\hline Hodgkin's lymphoma & $0 / 1$ & 33 & 9.7 \\
\hline Non-Hodgkin's lymphoma & $2 / 4$ & $67.5(52-93)$ & $5.4(1.4)$ \\
\hline Breast & $0 / 10$ & $63(47-74)$ & $4.0(0.6)$ \\
\hline Gastric & $1 / 0$ & 67 & 4.1 \\
\hline Melanoma & $1 / 1$ & $69(49-81)$ & $1.1(1.1)$ \\
\hline Pancreatic & $2 / 0$ & $74.5(74-75)$ & $24.3(2.7)$ \\
\hline Hepatocellular & $1 / 0$ & 46 & 3.8 \\
\hline Sarcoma & $1 / 0$ & 82 & 4.3 \\
\hline Adenoid cystic & $1 / 0$ & 48 & 4.9 \\
\hline Waldenstrom's & $0 / 1$ & 70 & 7.4 \\
\hline Unknown primary & $2 / 1$ & $72(70-74)$ & $7.4(1.0)$ \\
\hline All & 56 & $66(33-93)$ & $6.3(1.2)$ \\
\hline \multicolumn{4}{|l|}{ Peritoneal effusions and fluid } \\
\hline \multicolumn{4}{|l|}{ Mesothelioma } \\
\hline Epithelial & $1 / 1$ & $67(64-70)$ & $27.9(0.9)$ \\
\hline Cytology only & $5 / 0$ & $68(45-75)$ & $59.7(0.8)$ \\
\hline All & $1 / 6$ & $68(45-75)$ & $48.0(0.9)$ \\
\hline \multicolumn{4}{|c|}{ Non-mesothelioma malignant effusions } \\
\hline Ovarian cancer & $0 / 4$ & $65.5(54-70)$ & $73.7(0.8)$ \\
\hline Other cancer & $7 / 3$ & $62.5(48-76)$ & $3.6(0.7)$ \\
\hline Breast caner & $0 / 3$ & $66(48-76)$ & $5.0(0.6)$ \\
\hline Colon cancer & $5 / 0$ & $57(56-74)$ & $4.4(0.2)$ \\
\hline Lung cancer & $1 / 0$ & 69 & 2.9 \\
\hline Non-Hodgkin's lymphoma & $1 / 0$ & 59 & 0.6 \\
\hline \multicolumn{4}{|l|}{ Non-malignant effusions } \\
\hline Liver disease & $2 / 4$ & $57(32-73)$ & $3.0(0.6)$ \\
\hline \multicolumn{4}{|l|}{ Dialysis fluid } \\
\hline End-stage renal failure & $9 / 6$ & $64.5(36-85)$ & $0.2(1.1)$ \\
\hline
\end{tabular}

Of the two patients with lung cancer and mesothelin concentrations $>50 \mathrm{nM}$ in their effusions, one was diagnosed with bronchioalveolar carcinoma and the other with lung adenocarcinoma. Of the two patients with other malignancies and mesothelin concentrations $>50 \mathrm{nM}$, one was diagnosed with likely low-grade non-Hodgkin's lymphoma and the other with pancreatic carcinoma.

\section{Correlation of mesothelin levels in pleural effusions and serum}

In order to determine whether mesothelin concentrations in pleural effusions added diagnostic value to those in the serum, we compared mesothelin concentrations in matching fluid and serum samples from 41 patients with mesothelioma (fig 2A); 22 had increased mesothelin levels in both the effusion and in the serum, 2 had increased mesothelin levels in the serum only and 6 had raised mesothelin levels in the effusion but not in the serum. Of the 11 patients who were negative for mesothelin in the serum and the effusion, 5 were predominantly of the sarcomatoid variant. There was a significant correlation between mesothelin concentrations in the serum and pleural effusion $(\mathrm{p}<0.0001$; Pearson's correlation coefficient $0.67,95 \%$ CI 0.45 to 0.81 ).

Matching effusion and serum samples were available for 31 patients with non-mesothelioma conditions, 16 with benign effusions and 15 with malignant effusions (fig 2B). Four of the 15 patients with non-mesothelioma malignant effusions had increased mesothelin levels in the pleural effusion, and in 2 of these the mesothelin level was also raised in the serum. Three of these 15 patients had lung adenocarcinoma and the others had non-Hodgkin's lymphoma. The patient with raised mesothelin levels in the effusion but not in the serum had a diagnosis of pancreatic carcinoma. One patient with melanoma metastatic to the lungs had increased levels of mesothelin in the serum but not in the effusion. There was no correlation between serum and effusion mesothelin concentrations in patients with non-mesothelioma malignancy. 

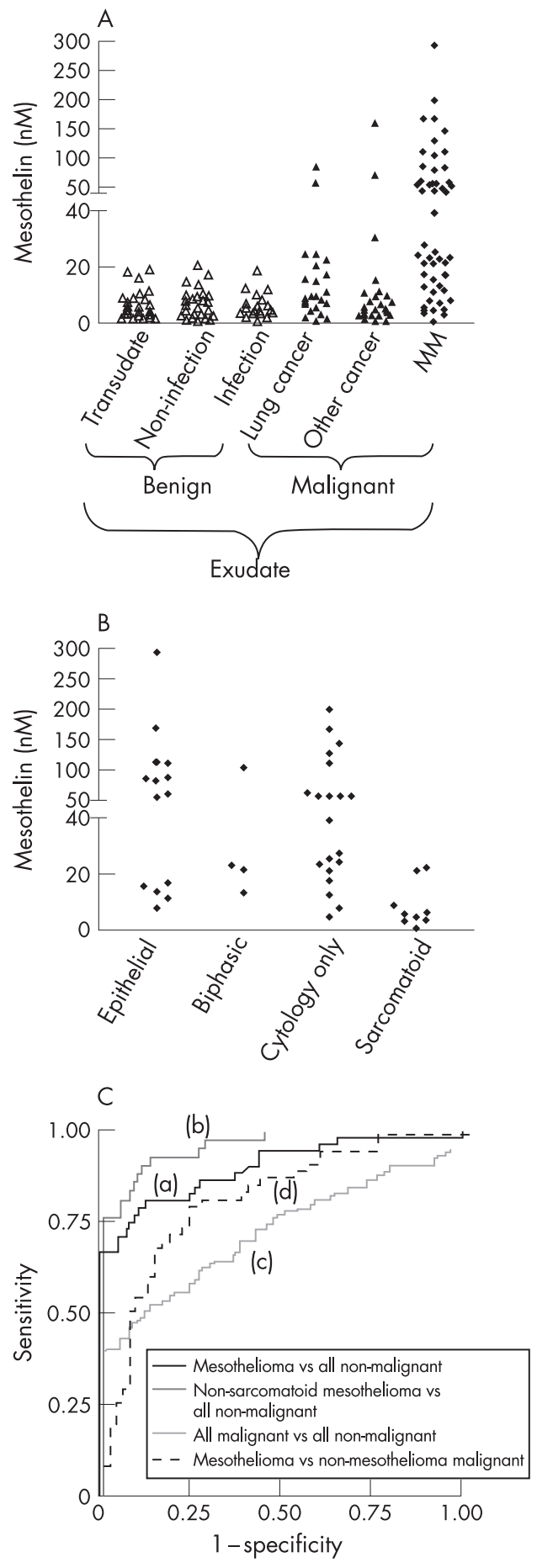

Figure 1 (A) Mesothelin concentrations in pleural effusions of patients, defined as transudate or exudate in nature and as benign or resulting from malignancy. Values were determined at least in duplicate by ELISA and individual patient values are plotted (B) Mesothelin concentrations in pleural effusions of patients with malignant mesothelioma further characterised by the histology of the tumour or where a diagnosis was made without histology (cytology only). (C) Receiver operating characteristic (ROC) curve showing accuracy of mesothelin effusion concentration in differentiating between (a) all patients with mesothelioma, (b) patients with epithelial, biphasic and cytologically defined mesothelioma and (c) all patients with malignancy from individuals with effusions of a non-malignant nature. Curve (d) shows the accuracy of mesothelin in differentiating between patients with mesothelioma and those with non-mesothelioma malignancies. Areas under the ROC curve were: (a) 0.898 (95\% Cl 0.839 to 0.958 ); (b) 0.964 (95\% Cl 0.934 to 0.994 ); (c) $0.748(95 \% \mathrm{Cl} 0.681$ to 0.816$)$ and (d) $0.896(95 \% \mathrm{Cl} 0.731$ to 0.896$)$. $\mathrm{MM}$, malignant mesothelioma.
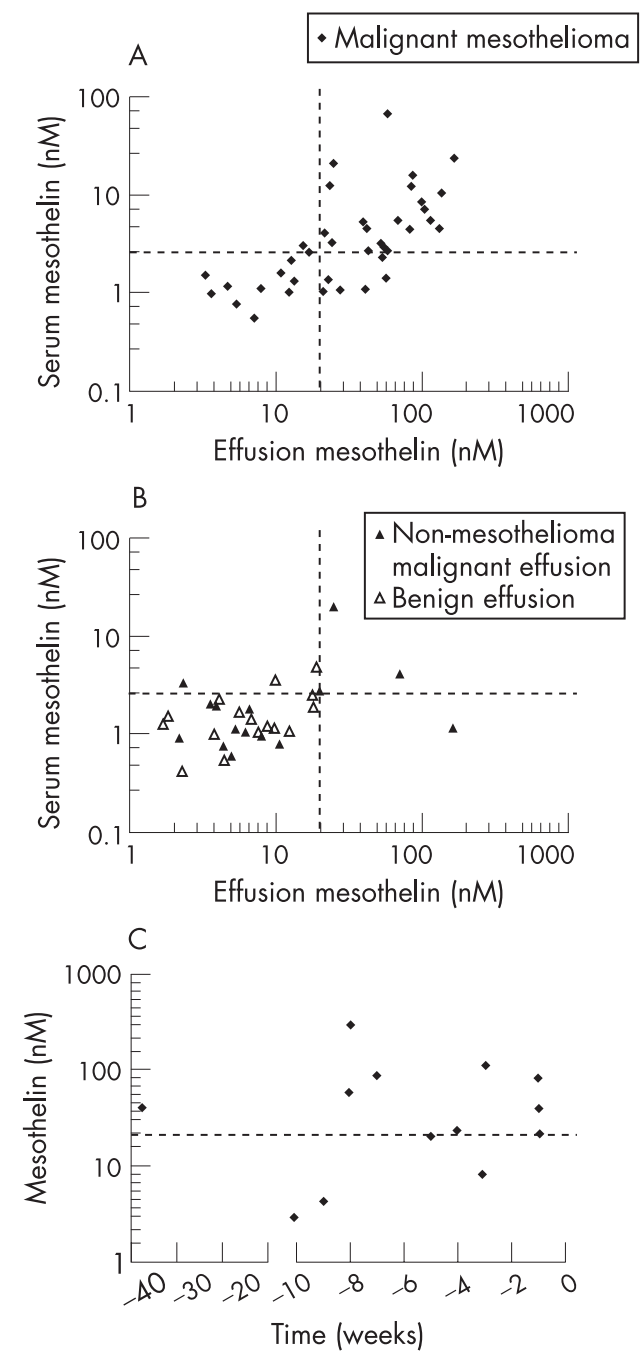

Figure 2 Correlation of mesothelin concentrations in serum and pleural effusions from (A) patients with mesothelioma and (B) patients with nonmesothelioma malignant conditions (solid triangle) and with benign conditions (open triangle). (C) Mesothelin concentrations in pleural effusions of patients with mesothelioma before pathological diagnosis of mesothelioma. A threshold value of $20 \mathrm{nM}$ for mesothelin concentrations in effusions and of $2.5 \mathrm{nM}$ in serum are indicated on the graph by dashed lines.

\section{Mesothelin levels in effusions at and before mesothelioma diagnosis}

Effusion samples were available from 13 individuals who were subsequently diagnosed with mesothelioma obtained I39 weeks before eventual diagnosis. No definite malignant cells were visible in these samples by cytological examination. The level of mesothelin was raised in 10 of these 13 prediagnosis samples (fig 2C). To determine if serum mesothelin levels alone would have made the diagnosis without the need to examine fluid mesothelin levels, serum mesothelin concentrations in patients with raised pre-diagnosis mesothelin levels in their fluid was determined; 4 of these patients had raised serum and effusion levels, 4 had raised levels in the effusion only, and 2 had no matching serum sample available.

\section{Relation of mesothelin levels in effusions with survival in patients with mesothelioma}

Survival data for patients with mesothelioma with mesothelin concentrations above the median level of the entire cohort (26 nM, table 1) were plotted against survival data for patients with mesothelin concentrations below the median and tested 


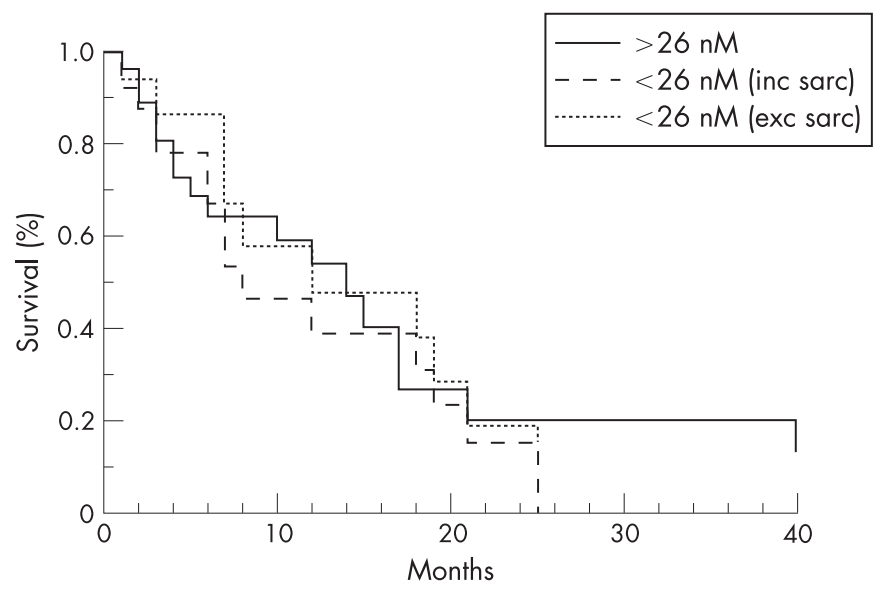

Figure 3 Median survival of patients with mesothelioma according to the mesothelin concentration in the first available pleural effusion sample for each individual.

for significant difference using the Kaplan-Meier method (fig 3). The median survival was 14 months for patients with high effusion levels of mesothelin $(n=27)$ and 8 months for those with low levels $(n=25)$. As patients with mesothelioma with sarcomatoid histology have a poorer prognosis than those with other histologies, we also examined the survival of patients with non-sarcomatoid mesothelioma with mesothelin concentrations below $26 \mathrm{nM}$. The median survival of this group of patients was 12 months $(n=16)$. There was no significant difference in survival of patients with mesothelioma in relation to the earliest available mesothelin effusion concentration using the Kaplan-Meier method or Cox's proportional hazards regression (data not shown).

\section{Mesothelin levels in peritoneal effusions and fluid}

Mesothelin concentrations above $20 \mathrm{nM}$ were found in 5 out of 7 patients with mesothelioma and in all 4 with ovarian cancer (median (SE) estimate 48 (0.9) nM and 73.7 (0.8) nM, respectively). All 10 patients with malignant effusions of other histologies and all non-malignant exudates were mesothelin

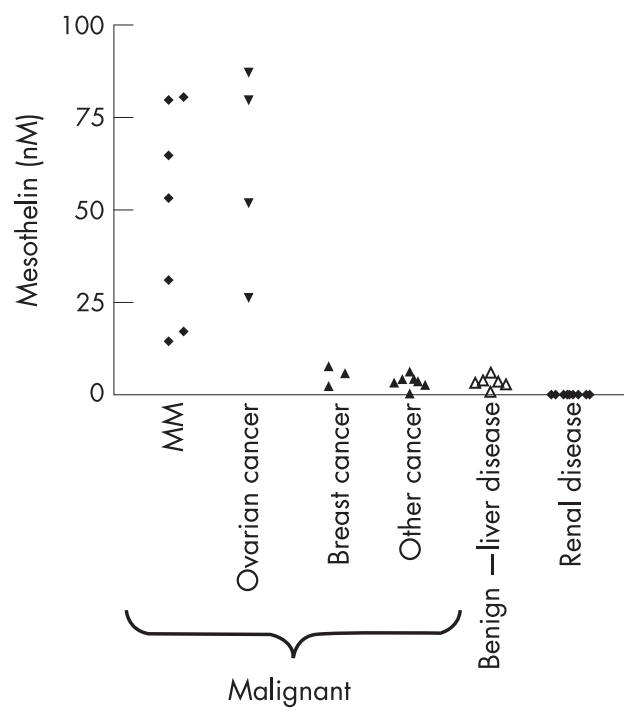

Figure 4 Mesothelin concentrations in peritoneal effusions from patients with malignant and benign disease, and in dialysis fluid from patients with renal failure. Mesothelin values were determined at least in duplicate by ELISA and individual patient values are plotted. MM, malignant mesothelioma. negative (median (SE) estimates $3.6 \quad(0.7) \mathrm{nM}$ and 3.0 (0.6) $\mathrm{nM}$, respectively). Mesothelin levels in continuous ambulatory peritoneal dialysis fluid from patients with chronic renal failure (in which large numbers of reactive mesothelial cells are usually found) had no detectable mesothelin (fig 4, table 1).

\section{Mesothelin staining}

Sarcomatous mesothelioma tissue does not stain with mesothelin (data not shown). To determine whether high or low mesothelin levels in the effusions of patients with nonsarcomatoid mesothelioma reflected the pattern of mesothelin expression in the tumour cells present in the effusion, immunohistochemical staining was done on a subset of samples from these patients (table 2, fig 5). Of the 6 samples from patients with mesothelin concentrations in the pleural effusion of $>26 \mathrm{nM}, 5$ stained positive for mesothelin and in the sixth sample the staining was equivocal. Mesothelin staining was predominantly membranous and may be associated with the microvilli. Of the 7 patients with pleural effusion concentrations of mesothelin $<26 \mathrm{nM}$, only two had positive staining for mesothelin and this was weak.

\section{DISCUSSION}

Given that an increased level of mesothelin in the serum is relatively specific and sensitive for mesothelioma, ${ }^{9}{ }^{10}$ and given that the majority of such patients present with exudative effusions, ${ }^{1}$ we have investigated whether mesothelin levels in effusions add value to the analysis of serum mesothelin levels. In this study we show that mesothelin levels above $20 \mathrm{nM}$ in effusions are highly suggestive of malignancy, particularly of mesothelioma, and that measurement of mesothelin levels in effusions could facilitate earlier diagnosis.

Several previous reports have described the use of tumour markers for the differential diagnosis of pleural effusions, but none have entered routine clinical use. ${ }^{216}{ }^{17}$ In the current study, effusion levels of mesothelin have a sensitivity of $67 \%$ at the high specificity of $98 \%$ for distinguishing mesothelioma from non-malignant effusions, and these levels of sensitivity and specificity indicate that this marker is useful in the investigation of patients with undiagnosed pleural or peritoneal effusions. The ROC curve generated for these data had an area under the curve of 0.898 (95\% CI 0.839 to 0.958 ), a result very similar to that reported by Scherpereel and colleagues who generated a ROC curve from patients in a multicentre study in France with an area under the curve of 0.831 (95\% CI 0.734 to 0.927). ${ }^{10}$ The concordance of the results from these independent studies is promising for the future use of mesothelin in diagnosing effusions. Furthermore, 15\% of non-mesothelioma tumours had increased effusion levels of mesothelin, suggesting that a mesothelin level above $20 \mathrm{nM}$ should be considered suspicious of malignancy, particularly (but not exclusively) malignant mesothelioma. Increased effusion levels of mesothelin in the absence of cytologically visible malignant cells suggests that mesothelin measurement should be performed when there is an index of suspicion of cancer, regardless of the absence of malignant cells.

The finding that some patients with non-mesothelioma malignant effusions had raised effusion levels of mesothelin is consistent with the fact that mesothelin staining has been reported in approximately $40 \%$ of lung and $85 \%$ of pancreatic adenocarcinomas. ${ }^{18}{ }^{19}$ To our knowledge, mesothelin expression has not been examined in non-Hodgkin's lymphoma and it is unclear why one of the six patients with this malignancy in the present study had raised mesothelin levels. It is noteworthy that a negative mesothelin result does not rule out malignancy. The biological reason why some patients with epithelial mesothelioma do not produce mesothelin is currently under 
Table 2 Correlation between mesothelin staining and mesothelin level in patients with mesothelioma

\begin{tabular}{llllll}
\hline Group & $\begin{array}{l}\text { Patient } \\
\text { number }\end{array}$ & Histology & $\begin{array}{l}\text { Effusion mesothelin } \\
\text { concentration (nM) }\end{array}$ & $\begin{array}{l}\text { Mesothelin } \\
\text { staining* }\end{array}$ & $\begin{array}{l}\text { Staining } \\
\text { intensity }\end{array}$ \\
\hline$<26 \mathrm{nM}$ & 1 & Mixed & 2 & - & + \\
& 2 & Cytology only & 12 & - & + \\
& 3 & Cytology only & 17 & + & + \\
& 4 & Cytology only & 21 & - & + \\
& 5 & Mixed & 22 & + & ++ \\
& 6 & Cytology only & 23 & + & + \\
& 7 & Cytology only & 25 & + & ++ \\
& 1 & Cytology only & 42 & + & ++ \\
& 2 & Cytology only & 52 & + & + \\
& 3 & Epithelial & 83 & + & + \\
\hline
\end{tabular}

E, equivocal

*A positive result was defined as the presence of membranous stain in more than $20 \%$ of tumour cells.

tStaining intensity was graded semi-quantitatively as negative, weak $(1+)$, moderate $(2+)$ or strong $(3+)$.
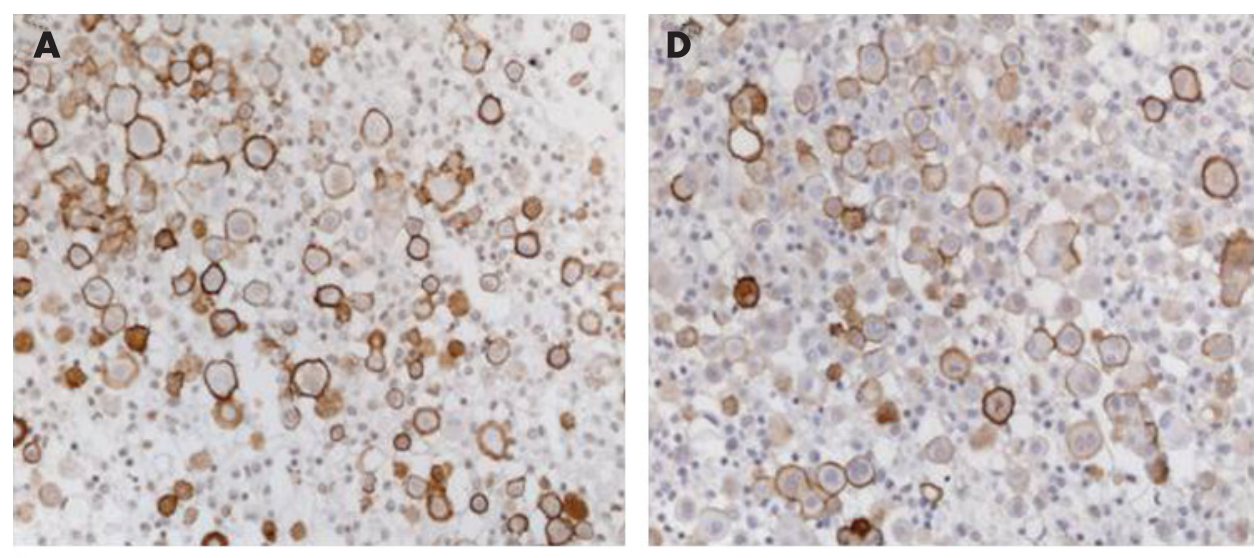

Figure 5 Immunostaining of sections of

formalin-fixed paraffin-embedded cell pellets from pleural fluid specimens of patients diagnosed with mesothelioma of unknown histology. (A, B) Cells from a sample with mesothelin concentration $52 \mathrm{nM}$. (C, D) Cells from a sample with mesothelin concentration $12 \mathrm{nM}$. (E, F) Cells from a sample with mesothelin concentration $23 \mathrm{nM}$. A, C and E stained with an antibody against mesothelin; $B, D$ and $F$ stained with an antibody against epithelial membrane antigen to demonstrate the presence of malignant mesothelioma cells.
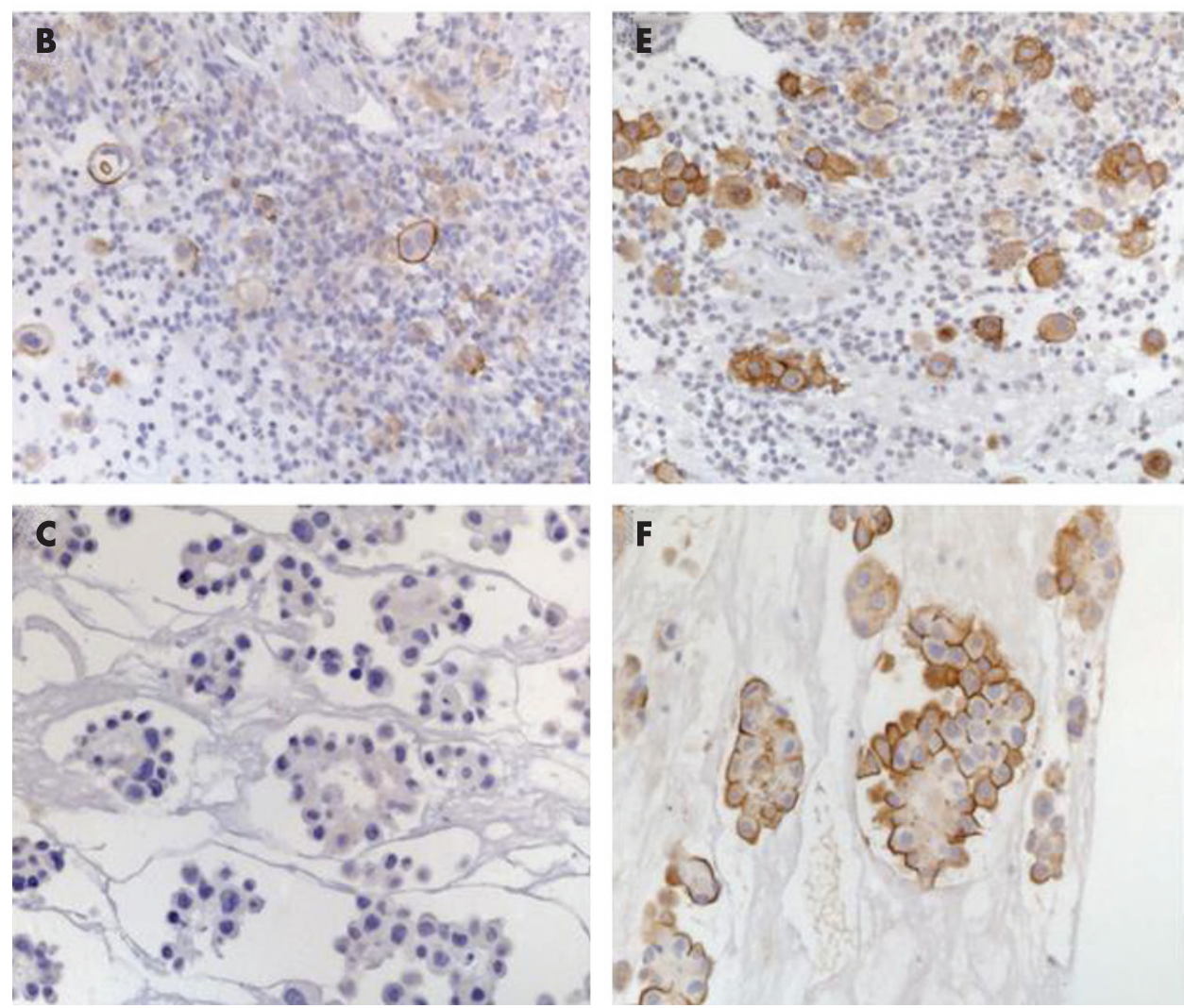
investigation. It is likely that the reason patients with sarcomatous mesothelioma have low levels of mesothelin in the serum and effusions is the lack of that protein in the tissue based on histological staining. ${ }^{20}$

As mesothelin is expressed on the surface of normal mesothelial cells lining the serosal cavities and can be enzymatically cleaved from the cell surface by trypsin or proteinase $\mathrm{K}^{21}$ it is conceivable that inflammatory conditions would produce raised mesothelin levels in effusions. The fact that mesothelin levels in exudative effusions, with or without infection, were not raised increases the likelihood that measurement of effusion mesothelin levels is a useful diagnostic test. This is in contrast to other markers such as CA125 and CA19-9 which are also released by normal mesothelial cells but are increased in inflammatory states, reducing their diagnostic specificity. ${ }^{2}$

It has not been possible to recover any pleural fluid from normal pleural cavities for analysis, so it is impossible to define a normal pleural mesothelin level. At a cut-off value of $20 \mathrm{nM}$ the specificity of mesothelin in non-malignant effusions was high (98\%), suggesting that mesothelin levels were not raised as a non-specific effect of inflammatory or reactive mesothelial processes.

The finding that mesothelin levels in effusions generally correlate with levels in the serum is to be expected, given the size of the molecule and the location of the tumour. However, mesothelin levels were increased before diagnosis in the effusions of 4 out of 8 patients when serum mesothelin levels were normal. The measurement of mesothelin in the fluid of patients with suspected mesothelioma may therefore prove to be of use even when serum mesothelin levels remain normal. Not all patients with mesothelioma present with an effusion, so the measurement of a serum biomarker such as mesothelin will be the only systemic aid for the diagnosis of this disease in such patients.

Increased effusion levels of mesothelin before a definitive cytological and/or histological diagnosis may provide an early suggestion of the presence of malignancy and indicate the need for active invasive investigation such as thoracoscopy to establish a diagnosis. Early diagnosis could reduce the costs of subsequent hospitalisations and investigations, reduce the "anxiety of not knowing" in patients who know they have a potentially malignant effusion (eg, those with substantial asbestos exposure whose colleagues may have died of mesothelioma) and provide the opportunity for early treatment using new treatment regimens which have been proved to be of some value..2 Whether early intervention achieves these goals is yet to be confirmed.

The current data indicate that mesothelin levels provide no prognostic information in patients with mesothelioma. This is predictable, given that a low concentration of mesothelin in an effusion may either reflect a small tumour burden (which may have a better prognosis) or a less differentiated tumour such as sarcomatoid mesothelioma (which has a worse prognosis). ${ }^{23}$

Patients with benign peritoneal effusions did not have increased levels of mesothelin, although benign peritoneal effusions are less frequent so are harder to obtain than benign pleural effusions. Mesothelin levels were not raised even in patients undergoing continuous ambulatory peritoneal dialysis in which a marked reactive mesothelial cell proliferation is characteristically seen in the peritoneal fluid. ${ }^{24}$ Although this confirms that reactive mesothelial cells in this clinical situation do not release large amounts of mesothelin into the fluid, it is possible that mesothelin protein would not have had time to accumulate into the dialysate or, indeed, if the dialysate dilutes mesothelin concentrations beyond the detection levels of this assay. Patients with ovarian cancer, which has a number of biological similarities to mesothelioma and who also exhibit raised serum levels of mesothelin, ${ }^{25}$ also exhibit raised levels of mesothelin in the peritoneal fluid. None of the patients studied had raised levels of mesothelin in the peritoneal fluid, although not all possible cancer types that can involve the peritoneum have been studied to date. It appears that the value of measuring mesothelin in peritoneal fluid is similar to that in pleural fluid for patients with suspected mesothelioma or ovarian carcinoma.

The correlation found between tissue mesothelin expression and pleural fluid mesothelin levels suggests that the tumour (or the tumour microenvironment) is responsible for producing the mesothelin protein. This could be by cleavage of surface-bound mesothelin ${ }^{2627}$ by tumour proteases or by generation of a soluble form of mesothelin by alternative splicing of the mesothelin mRNA. ${ }^{25}$ As the antibodies in the mesothelin assay have the potential to react with both surface-bound and soluble mesothelin, this study does not directly resolve this issue. Given that mesothelin staining was observed on the surface of mesothelioma cells present in two of seven pleural effusions with low mesothelin concentrations, both scenarios are possible. The absence of increased fluid levels of mesothelin in inflammatory states suggests that it is not easily cleaved from the surface and that there is something particular about the malignant state that generates high mesothelin levels in effusions.

Other markers for mesothelioma are currently under study (eg, osteopontin ${ }^{28}$ and RCASl, a type II membrane protein ${ }^{29}$ ). Mesotheliomas of sarcomatoid histology stain positively for RCAS but, in the small sample set published, levels in effusions were not increased compared with those in lung cancer. ${ }^{29}$ Given that a panel of tumour markers would improve specificity and specificity, ${ }^{316}$ further work is required to determine which of these and other markers could improve the sensitivity and specificity of mesothelin in diagnosing mesothelioma.

Mesothelin measurements in serum are now undertaken in a number of centres in the world as an aid to the diagnosis of mesothelioma using a standardised commercially available ELISA assay. Until now the measurement of tumour markers in pleural effusions has not been part of routine clinical practice. ${ }^{17}$ The data presented here argue that measurement of mesothelin in effusions (pleural or peritoneal) might be a useful adjunct to serum analysis in patients with suspected malignancy, particularly if the index of suspicion for mesothelioma is high. As effusion fluid is routinely sent for pathological, biochemical and often microbial analysis, it is a simple matter to undertake mesothelin analysis at the same time. Clearly, in cases where fluid cytology shows mesothelioma cells with a high degree of certainty, measurement of mesothelin would add little; however, a high level of mesothelin in the fluid, especially in the absence of malignant cells, suggests a diagnosis of malignancy, particularly mesothelioma, and the need for early biopsy. The availability of this simple test may be particularly useful in centres not experienced in the cytopathological diagnosis of this disease and where diagnosis is often complicated and time consuming.

\section{ACKNOWLEDGEMENTS}

The authors thank the staff of PathWest Laboratory Medicine, Sir Charles Gairdner Hospital, Hollywood Hospital and St John of God Pathology for their assistance with this study and acknowledge the assistance and advice of Judy Innes-Rowe, Nola Olsen, Michael Platen and Steve Fletcher.

\footnotetext{
Authors' affiliations

Jenette Creaney, Deborah Yeoman, Leanne K Naumoff, Michelle Hof, Arthur William Musk, Nicholas De Klerk, Bruce W S Robinson, National Research Centre for Asbestos Related Diseases, Western Australian Institute of Medical Research, University of Western Australia, Sir Charles Gairdner Hospital, Perth, Australia
} 
Jenette Creaney, Michelle Hof, Bruce W S Robinson, Australian

Mesothelioma Tissue Bank, Sir Charles Gairdner Hospital, Perth, Australia Amanda Segal, PathWest Laboratory Medicine WA, Queen Elizabeth II Medical Centre, Perth, Australia

Arthur William Musk, Department of Respiratory Medicine, Sir Charles Gairdner Hospital, Perth, Australia

Nicholas De Klerk, Telethon Institute of Child Health Research, Subiaco, AustraliaBiostatistics Center, Massachusetts General Hospital and Harvard Medical School, Boston, Massachusetts, USA

Supported in part by research grants from the National Health and Medical Research Council of Australia and the Insurance Commission of Western Australia, the Raine Foundation, and in part by Fujirebio Diagnositic Malvern, Pennsylvania, USA. Biospecimens were provided by members of the $A B N$-Oncology Group which is funded by the National Health and Medical Research Council of Australia.

JC and BWR have received consultancy fees from FDI. The remaining authors have no conflicts of interest to disclose.

\section{REFERENCES}

1 Robinson BW, Musk AW, Lake RA. Malignant mesothelioma. Lancet 2005;366:397-408.

2 Shitrit $D$, Zingerman $B$, Shitrit $A B$, et al. Diagnostic value of CYFRA 21-1, CEA, CA 19-9, CA 15-3, and CA 125 assays in pleural effusions: analysis of 116 cases and review of the literature. Oncologist 2005;10:501-7.

3 Villena V, Lopez-Encuentra A, Echave-Sustaeta J, et al. Diagnostic value of CA 549 in pleural fluid. Comparison with CEA, CA 15.3 and CA 72.4. Lung Cancer 2003;40:289-94.

4 Miedouge M, Rouzaud P, Salama G, et al. Evaluation of seven tumour markers in pleural fluid for the diagnosis of malignant effusions. Br J Cancer 1999;81:1059-65.

5 Alatas F, Alatas O, Metintas M, et al. Diagnostic value of CEA, CA 15-3, CA 199, CYFRA 21-1, NSE and TSA assay in pleural effusions. Lung Cancer 2001;31:9-16.

6 Mezger J, Calavrezos A, Drings $P$, et al. Value of serum and effusion fluid CEA levels for distinguishing between diffuse malignant mesothelioma and carcinomatous pleural metastases. Lung 1994;172:183-4.

7 Fuhrman C, Duche JC, Chouaid C, et al. Use of tumor markers for differential diagnosis of mesothelioma and secondary pleural malignancies. Clin Biochem 2000:33:405-10.

8 Paganuzzi M, Onetto M, Marroni P, et al. Diagnostic value of CYFRA 21-1 tumor marker and CEA in pleural effusion due to mesothelioma. Chest $2001 ; 119: 1138-42$.

9 Robinson BW, Creaney J, Lake R, et al. Mesothelin-family proteins and diagnosis of mesothelioma. Lancet 2003;362:1612-6.
10 Scherpereel A, Grigoriu BD, Conti M, et al. Soluble mesothelin-related peptides in the diagnosis of malignant pleural mesothelioma. Am J Respir Crit Care Med 2006;173:1155-60.

11 Creaney J, Christansen H, Lake R, et al. Soluble mesothelin related protein in mesothelioma. J Thorac Oncol 2006;1:172-4.

12 Light RW, Macgregor MI, Luchsinger PC, et al. Pleural effusions: the diagnostic separation of transudates and exudates. Ann Intern Med 1972;77:507-13.

13 Golub G, Heath M, Wahba G. Cross-validation and optimum ridge regression. Soc Ind Appl Math Rev 1976;18:806.

14 Cox D. Regression models and life-tables. J R Stat Soc: Series B Stat Method 1972;34:187.

15 Whitaker D. The cytology of malignant mesothelioma. Cytopathology 2000;1 1:139-51.

16 Porcel JM, Vives M, Esquerda A, et al. Use of a panel of tumor markers (carcinoembryonic antigen, cancer antigen 125, carbohydrate antigen 15-3, and cytokeratin 19 fragments) in pleural fluid for the differential diagnosis of benign and malignant effusions. Chest 2004;126:1757-63.

17 Light RW. Tumor markers in undiagnosed pleural effusions. Chest 2004;126:1721-2.

18 Ordonez NG. The immunohistochemical diagnosis of mesothelioma: a comparative study of epithelioid mesothelioma and lung adenocarcinoma. Am J Surg Pathol 2003;27:1031-51.

19 Ordonez NG. Application of mesothelin immunostaining in tumor diagnosis. Am J Surg Pathol 2003;27:1418-28.

20 Ordonez NG. Value of mesothelin immunostaining in the diagnosis of mesothelioma. Mod Pathol 2003;16:192-7.

21 Chang K, Pastan I, Willingham MC. Isolation and characterization of a monoclonal antibody, $\mathrm{Kl}$, reactive with ovarian cancers and normal mesothelium. Int J Cancer 1992;50:373-81.

22 Robinson BW, Lake RA. Advances in malignant mesothelioma. N Engl J Med 2005;353:1591-603

23 Segal A, Whitaker D, Henderson DW, et al. Pathology of mesothelioma. In: Robinson BWS, CHahinian PA, eds. Mesothelioma. London: Martin Dunitz, 2002: 143-84.

24 Rapoport J, Hausmann MJ, Chaimovitz C. The peritoneal immune system and continuous ambulatory peritoneal dialysis. Nephron 1999;81:373-80.

25 Scholler N, Fu N, Yang Y, et al. Soluble member(s) of the mesothelin/ megakaryocyte potentiating factor family are detectable in sera from patients with ovarian carcinoma. Proc Natl Acad Sci USA 1999;96:11531-6.

26 Hellstrom I, Raycraft J, Kanan S, et al. Mesothelin variant 1 is released from tumor cells as a diagnostic marker. Cancer Epidemiol Biomarkers Prev 2006;15:1014-20.

27 Ho M, Onda M, Wang QC, et al. Mesothelin is shed from tumor cells. Cancer Epidemiol Biomarkers Prev 2006;15:1751

28 Pass HI, Lott D, Lonardo F, et al. Asbestos exposure, pleural mesothelioma, and serum osteopontin levels. N Engl J Med 2005;353:1564-73.

29 Hiraki A, Aoe K, Murakami T, et al. Clinical significance of the expression of tumor-associated antigen, RCAS1, and its soluble protein in pleural fluid in malignant mesothelioma. Oncol Rep 2005; 14:357-62.

\section{Save your favourite articles and useful searches}

Use the "My folders" feature to save and organise articles you want to return to quickly-saving space on your hard drive. You can also save searches, which will save you time. You will only need to register once for this service, which can be used for this journal or all BMJ Journals, including the BMJ. 